\title{
Corrigendum
}

\section{Immunoprotective effects of oral intake of heat-killed Lactobacillus pentosus strain b240 in elderly adults: a randomised, double-blind, placebo-controlled trial - CORRIGENDUM}

Shoji Shinkai, Masamichi Toba, Takao Saito, Ikutaro Sato, Mina Tsubouchi, Kiyoto Taira, Keiji Kakumoto, Takashi Inamatsu, Hiroto Yoshida, Yoshinori Fujiwara, Taro Fukaya, Tetsuya Matsumoto, Kazuhiro Tateda, Keizo Yamaguchi, Noriyuki Kohda and Shigeru Kohno

(First published online 29 July 2016)

DOI: 10.1017/S0007114512003753. Published by Cambridge University Press, 5 September 2012

The authors regret to announce that there were some errors in Table 3 of this paper, namely that the number of subjects and the statistical analytical method for QOLs were incorrect. The corrected Table 3 and texts are below. We apologize for any inconvenience caused.

Original text and correction

\section{Abstract (P 1856, L11-12)}

General health perception, as determined by the SF-36®, dose-dependently increased in the b240 groups $(\boldsymbol{P}$ for trend $=\mathbf{0} \cdot \mathbf{0 1 6})$. (Correction)

General health perception, as determined by the SF-36®, dose-dependently increased in the b240 groups $(\boldsymbol{P}<\mathbf{0 \cdot 0 2 5})$

\section{Experimental methods (Statistical analysis) (P 1859, L13-19 of the right column)}

Dunnett's multiple comparison test was performed for the inter-group comparison of QOL score. To examine whether the effect of b240 on QOL score was independent of common cold experience, we used generalized linear models in which we entered changes in score ( $\Delta$ post - pre) as the target variable, and group (placebo to high-dose) and common cold experience (yes or no) as explanatory variables.

(Correction)

Shirley-Williams' multiple comparison test was performed for the inter-group comparison of QOL score. To examine whether the effect of b240 on QOL score was independent of common cold experience, we used generalized linear models in which we entered changes in score $\Delta$ (post - pre) as the target variable, and group (placebo to high-dose) and common cold experience (yes or no) as explanatory variables.

\section{Results (Change in quality of life) (P 1862, L2-6 of the left column)}

Table 3 shows a comparison of pre- and post-intervention QOL scores. From pre- to post-intervention, the score for the general health perception subscale in the b240 groups significantly improved as compared with that in the placebo group $(\boldsymbol{P}$ for trend $=\mathbf{0} \cdot \mathbf{0 4 1})$

(Correction)

Table 3 shows a comparison of pre- and post-intervention QOL scores. From pre- to post-intervention, the score for the general health perception subscale in the b240 groups significantly improved as compared with that in the placebo group $(\boldsymbol{P}<\mathbf{0 \cdot 0 2 5})$. 


\section{Table3. (P 1862)}

Table 3. Changes in quality of life as assessed by the SF-36 (per-protocol set) (Mean values and standard deviations)

\begin{tabular}{|c|c|c|c|c|c|c|c|c|c|}
\hline \multirow[b]{3}{*}{ Subscales of SF-36 } & \multirow[b]{3}{*}{ Groups } & \multirow[b]{3}{*}{$n$} & \multicolumn{6}{|c|}{ NBS score } & \multirow[b]{3}{*}{$P+$} \\
\hline & & & \multicolumn{2}{|c|}{ Pre-intervention } & \multicolumn{2}{|c|}{ Post-intervention } & \multicolumn{2}{|c|}{$\Delta(\text { post }- \text { pre })^{\star}$} & \\
\hline & & & Mean & SD & Mean & SD & Mean & SD & \\
\hline \multirow[t]{3}{*}{ Physical functioning } & Placebo & 93 & 51.4 & $7 \cdot 3$ & $50 \cdot 3$ & $9 \cdot 6$ & $-1 \cdot 1$ & $7 \cdot 1$ & Reference \\
\hline & Low-dose b240 & 92 & $52 \cdot 1$ & $5 \cdot 9$ & $50 \cdot 9$ & $7 \cdot 8$ & $-1 \cdot 2$ & $7 \cdot 1$ & 0.99 \\
\hline & High-dose b240 & 93 & $51 \cdot 2$ & 10.4 & $49 \cdot 7$ & $9 \cdot 3$ & -1.5 & $8 \cdot 1$ & 0.89 \\
\hline \multirow[t]{3}{*}{ Role physical } & Placebo & $\overline{93}$ & $52 \cdot 0$ & $7 \cdot 3$ & 49.5 & 11.0 & $-2 \cdot 4$ & $10 \cdot 8$ & Reference \\
\hline & Low-dose b240 & 92 & $52 \cdot 1$ & $6 \cdot 3$ & $50 \cdot 4$ & $8 \cdot 8$ & $-1 \cdot 6$ & $7 \cdot 6$ & 0.78 \\
\hline & High-dose b240 & 93 & $51 \cdot 6$ & $8 \cdot 7$ & $50 \cdot 4$ & $9 \cdot 4$ & $-1 \cdot 2$ & $9 \cdot 1$ & $\overline{0.56}$ \\
\hline \multirow[t]{3}{*}{ Body pain } & Placebo & 93 & 51.0 & $8 \cdot 3$ & $51 \cdot 0$ & 9.9 & 0.0 & 9.6 & Reference \\
\hline & Low-dose b240 & 92 & $53 \cdot 7$ & $8 \cdot 7$ & $52 \cdot 2$ & $11 \cdot 4$ & -1.5 & $9 \cdot 1$ & 0.48 \\
\hline & High-dose b240 & 93 & $52 \cdot 0$ & $8 \cdot 9$ & $51 \cdot 9$ & $9 \cdot 9$ & $-0 \cdot 1$ & $8 \cdot 8$ & $1 \cdot 00$ \\
\hline \multirow[t]{3}{*}{ General health perception } & Placebo & $\overline{93}$ & 54.9 & $7 \cdot 8$ & $53 \cdot 4$ & $8 \cdot 6$ & $-1 \cdot 4$ & $7 \cdot 4$ & Reference \\
\hline & Low-dose b240 & 92 & $54 \cdot 3$ & $7 \cdot 2$ & $54 \cdot 9$ & $9 \cdot 6$ & 0.7 & $8 \cdot 2$ & 0.11 \\
\hline & High-dose b240 & 93 & $55 \cdot 5$ & $7 \cdot \overline{8}$ & $56 \cdot 6$ & $8 \cdot 2$ & $1 \cdot 1$ & $6 . \overline{5}$ & 0.04 \\
\hline \multirow[t]{3}{*}{ Vitality } & Placebo & $\overline{93}$ & $57 \cdot 1$ & $7 \cdot 4$ & $55 \cdot 1$ & $9 \cdot \overline{6}$ & $-2 \cdot 0$ & 8.4 & Reference \\
\hline & Low-dose b240 & 92 & $57 \cdot 8$ & $7 \cdot 9$ & $55 \cdot 4$ & $8 \cdot 7$ & $-2 \cdot 4$ & $7 \cdot 2$ & 0.92 \\
\hline & High-dose b240 & 93 & 57.9 & $7 \cdot 1$ & $55 \cdot 9$ & 9.5 & $-2 \cdot 0$ & $6 \cdot 7$ & 1.00 \\
\hline \multirow[t]{3}{*}{ Social functioning } & Placebo & 93 & $52 \cdot 4$ & $7 \cdot 0$ & $52 \cdot 1$ & $8 \cdot 0$ & -0.3 & $10 \cdot 2$ & Reference \\
\hline & Low-dose b240 & 92 & $53 \cdot 6$ & $6 \cdot 9$ & $53 \cdot 1$ & $7 \cdot 0$ & -0.6 & $8 \cdot 2$ & 0.98 \\
\hline & High-dose b240 & 93 & 52.5 & $6 \cdot 8$ & $51 \cdot 1$ & 8.6 & $-1 \cdot 4$ & $9 \cdot \overline{7}$ & 0.67 \\
\hline \multirow[t]{3}{*}{ Role emotional } & Placebo & 93 & $52 \cdot 7$ & 6.9 & $51 \cdot 3$ & $9 \cdot 7$ & -1.5 & $10 \cdot 0$ & Reference \\
\hline & Low-dose b240 & 92 & $53 \cdot 6$ & $5 \cdot 2$ & $51 \cdot 4$ & $8 \cdot 4$ & $-2 \cdot 1$ & 6.8 & 0.82 \\
\hline & High-dose b240 & 93 & $52 \cdot 5$ & $7 \cdot 9$ & $51 \cdot 2$ & $9 \cdot 8$ & $-1 \cdot 3$ & $7 \cdot 9$ & 0.98 \\
\hline \multirow[t]{3}{*}{ Mental health } & Placebo & 93 & 54.9 & $7 \cdot 1$ & $55 \cdot 1$ & $7 \cdot 3$ & 0.2 & $7 \cdot 6$ & Reference \\
\hline & Low-dose b240 & 92 & $56 \cdot 0$ & 8.4 & $55 \cdot 0$ & $8 \cdot 7$ & -1.0 & $7 \cdot 2$ & 0.40 \\
\hline & High-dose b240 & 93 & $55 \cdot 8$ & $7 \cdot 1$ & $55 \cdot 7$ & $7 \cdot 2$ & -0.1 & $6 \cdot \overline{3}$ & 0.93 \\
\hline
\end{tabular}

NBS, norm-based scoring for Japanese; b240, Lactobacillus pentosus strain b240.

* Change in score was derived by subtracting pre-value from the post-value.

† Dunnett's multiple comparison test.

\section{(Correction)}

Table 3. Changes in quality of life as assessed by the SF-36 (per-protocol set) (Mean values and standard deviations)

\begin{tabular}{|c|c|c|c|c|c|c|c|c|c|}
\hline \multirow[b]{3}{*}{ Subscales of SF-36 } & \multirow[b]{3}{*}{ Groups } & \multirow[b]{3}{*}{$n$} & \multicolumn{6}{|c|}{ NBS score } & \multirow[b]{3}{*}{$P+$} \\
\hline & & & \multicolumn{2}{|c|}{ Pre-intervention } & \multicolumn{2}{|c|}{ Post-intervention } & \multicolumn{2}{|c|}{$\Delta(\text { post }- \text { pre })^{\star}$} & \\
\hline & & & Mean & SD & Mean & SD & Mean & SD & \\
\hline \multirow[t]{3}{*}{ Physical functioning } & Placebo & 93 & 51.4 & $7 \cdot 3$ & $50 \cdot 3$ & $9 \cdot 6$ & $-1 \cdot 1$ & $7 \cdot 1$ & Reference \\
\hline & Low-dose b240 & 92 & $52 \cdot 1$ & $5 \cdot 9$ & $50 \cdot 9$ & $7 \cdot 8$ & $-1 \cdot 2$ & $7 \cdot 1$ & NS \\
\hline & High-dose b240 & 92 & $51 \cdot 2$ & $10 \cdot 4$ & $49 \cdot 7$ & $9 \cdot 3$ & -1.5 & $8 \cdot 1$ & NS \\
\hline \multirow[t]{3}{*}{ Role physical } & Placebo & $\overline{93}$ & $52 \cdot 0$ & $7 \cdot 3$ & $49 \cdot 5$ & $11 \cdot 0$ & $-2 \cdot 4$ & $10 \cdot 8$ & Reference \\
\hline & Low-dose b240 & 92 & $52 \cdot 1$ & $6 \cdot 3$ & $50 \cdot 4$ & 8.8 & $-1 \cdot 6$ & $7 \cdot 6$ & NS \\
\hline & High-dose b240 & 93 & 51.6 & $8 \cdot 7$ & $50 \cdot 4$ & $9 \cdot 4$ & $-1 \cdot 2$ & $9 \cdot 1$ & NS \\
\hline \multirow[t]{3}{*}{ Body pain } & Placebo & 93 & $51 \cdot 0$ & $8 \cdot 3$ & $51 \cdot 0$ & 9.9 & 0.0 & $9 \cdot 6$ & Reference \\
\hline & Low-dose b240 & 92 & $53 \cdot 7$ & $8 \cdot 7$ & $52 \cdot 2$ & 11.4 & -1.5 & $9 \cdot 1$ & NS \\
\hline & High-dose b240 & 92 & $52 \cdot 0$ & $8 \cdot 9$ & $51 \cdot 9$ & $9 \cdot 9$ & $-0 \cdot 1$ & $8 \cdot 8$ & NS \\
\hline \multirow[t]{3}{*}{ General health perception } & Placebo & $\overline{93}$ & $54 \cdot 9$ & $7 \cdot 8$ & $53 \cdot 4$ & 8.6 & $-1 \cdot 4$ & $7 \cdot 4$ & Reference \\
\hline & Low-dose b240 & 92 & $54 \cdot 3$ & $7 \cdot 2$ & 54.9 & $9 \cdot 6$ & 0.7 & $8 \cdot 2$ & $<0.025$ \\
\hline & High-dose b240 & 92 & $55 \cdot 5$ & $7 \cdot 8$ & $56 \cdot 6$ & $8 \cdot 2$ & $1 \cdot 1$ & $6 \cdot 5$ & $<0.025$ \\
\hline \multirow[t]{3}{*}{ Vitality } & Placebo & $\overline{93}$ & $57 \cdot 1$ & $7 \cdot 4$ & $55 \cdot 1$ & $9 \cdot 6$ & $-2 \cdot 0$ & $8 \cdot 4$ & Reference \\
\hline & Low-dose b240 & 92 & $57 \cdot 8$ & $7 \cdot 9$ & $55 \cdot 4$ & $8 \cdot 7$ & $-2 \cdot 4$ & $7 \cdot 2$ & NS \\
\hline & High-dose b240 & 93 & $57 \cdot 9$ & $7 \cdot 1$ & $55 \cdot 9$ & 9.5 & $-2 \cdot 0$ & $6 \cdot 7$ & NS \\
\hline \multirow[t]{3}{*}{ Social functioning } & Placebo & 93 & $52 \cdot 4$ & $7 \cdot 0$ & $52 \cdot 1$ & 8.0 & -0.3 & $10 \cdot 2$ & Reference \\
\hline & Low-dose b240 & 92 & $53 \cdot 6$ & 6.9 & $53 \cdot 1$ & $7 \cdot 0$ & -0.6 & 8.2 & NS \\
\hline & High-dose b240 & 93 & 52.5 & $6 \cdot 8$ & $51 \cdot 1$ & 8.6 & -1.4 & 9.7 & NS \\
\hline \multirow[t]{3}{*}{ Role emotional } & Placebo & 93 & $52 \cdot 7$ & $6 \cdot 9$ & $51 \cdot 3$ & $9 \cdot 7$ & -1.5 & $10 \cdot 0$ & Reference \\
\hline & Low-dose b240 & 92 & $53 \cdot 6$ & $5 \cdot 2$ & 51.4 & $8 \cdot 4$ & $-2 \cdot 1$ & $6 \cdot 8$ & NS \\
\hline & High-dose b240 & 93 & $52 \cdot 5$ & $7 \cdot 9$ & $51 \cdot 2$ & $9 \cdot 8$ & $-1 \cdot 3$ & $7 \cdot 9$ & NS \\
\hline \multirow[t]{3}{*}{ Mental health } & Placebo & 93 & $54 \cdot 9$ & $7 \cdot 1$ & $55 \cdot 1$ & $7 \cdot 3$ & 0.2 & $7 \cdot 6$ & Reference \\
\hline & Low-dose b240 & 92 & $56 \cdot 0$ & $8 \cdot 4$ & $55 \cdot 0$ & $8 \cdot 7$ & $-1 \cdot 0$ & $7 \cdot 2$ & NS \\
\hline & High-dose b240 & 93 & $55 \cdot 8$ & $7 \cdot 1$ & $55 \cdot 7$ & $7 \cdot 2$ & -0.1 & $6 \cdot 3$ & NS \\
\hline
\end{tabular}

NBS, norm-based scoring for Japanese; b240, Lactobacillus pentosus strain b240.

* Change in score was derived by subtracting pre-value from the post-value.

† Shirley-Williams' multiple comparison test (one - side, $2.5 \%$ ). NS not significant. 


\section{Reference}

Shinkai S, Toba M, Saito T, et al. (2013) Immunoprotective effects of oral intake of heat-killed Lactobacillus pentosus strain b240 in elderly adults: a randomised, double-blind, placebo-controlled trial. Br J Nutr 109, 1856-1865. doi:10.1017/S0007114512003753. 